\title{
Numerical Models of Skin Electropermeabilization Taking Into Account Conductivity Changes and the Presence of Local Transport Regions
}

\author{
Nataša Pavšelj and Damijan Miklavčič
}

\begin{abstract}
The protective function of skin (its low permeability) presents a formidable obstacle in therapeutical applications such as transdermal drug delivery and gene delivery in skin. One of the possibilities to temporarily breach the barrier function of skin is using electroporation, creating aqueous pathways across lipid-based structures by means of electric pulses. In addition, the application of electric pulses to biological cells causes the electroporation of cell membrane, increasing its permeability, thus enabling cell uptake of larger molecules that otherwise cannot cross the membrane, such as drug molecules or DNA. The electropermeabilization process in skin was described theoretically, by means of numerical modeling, leaning on data derived from our in vivo experiments previously published. The numerical models took into account the layered structure of skin, macroscopical changes of its bulk electrical properties during electroporation, as well as the presence of localized sites of increased molecular transport termed local transport regions. The output of the models was compared with the in vivo experiments, and a good agreement was obtained. In addition, a comparison of our results with already published findings on skin electropermeabilization showed that permeabilizing voltage amplitudes suggested by the model are also well in the range of the voltage amplitudes reported by other authors to cause skin permeabilization. The subject of tissue conductivity changes due to electroporation is still a rather unexplored field; however, we used the available data to describe the mechanism of the nonlinear process of the tissue electropermeabilization.
\end{abstract}

Index Terms-Conductivity changes, finite element method, local transport regions (LTRs), numerical modeling, skin electroporation.

\section{INTRODUCTION}

$\mathbf{S}$ KIN COVERS the entire external surface of the human body, is the principal site of interaction with the surrounding world, and serves as the body's protective barrier. Due to its size and accessibility, skin is an attractive target tissue for a variety of applications. Most notably, transdermal drug delivery is an approach used to deliver drugs into the skin for therapeutic use by means of chemical and/or physical enhancers [1], [2] as an alternative to oral, intravascular, subcutaneous, and transmu-

Manuscript received October 30, 2007; revised January 19, 2008. This work was supported in part by the European Commission under the FP5 Grant CLINIPORATOR (QLK-1999-00484) and under the FP6 Grant ANGIOSKIN (LSHB-CT-2005-512127) and in part by the Slovenian Research Agency.

The authors are with the Faculty of Electrical Engineering, University of Ljubljana, 1000 Ljubljana, Slovenia (e-mail: natasa.pavselj@fe.uni-lj.si; damijan.miklavcic@fe.uni-lj.si).

Color versions of one or more of the figures in this paper are available online at http://ieeexplore.iee.org.

Digital Object Identifier 10.1109/TPS.2008.928715 cosal routes. Skin is also an attractive target tissue for in vivo gene delivery [3], [4]. However, the protective function of skin (its low permeability) presents a formidable obstacle and limits the number of drugs that can be delivered transdermally. One of the possibilities to temporarily breach the barrier function of skin is using electroporation, thereby creating aqueous pathways across lipid-based structures [5], [6]. Electroporation is a phenomenon in which the cell membranes exposed to highvoltage electric pulses of adequate amplitude and duration are temporarily destabilized and permeabilized. The increase in permeability of the cell membrane makes it possible for larger molecules that otherwise cannot cross the membrane, such as drug molecules or DNA, to enter the cell [7]. After pulsing, the cell membrane reseals, provided that the applied voltage was not too high to cause permanent cell membrane damage. In this paper, the electropermeabilization process in skin is described theoretically, by means of numerical modeling, based on data derived from in vivo experiments resulting from our previous research [8] and the literature [9]-[19].

When referring to its electrical properties, skin is a very intricate tissue due to its highly inhomogeneous structure. Skin epidermis contains different layers, but the one that defines its electrical properties the most is the outermost layer, the stratum corneum, composed of dead flat skin cells. Although very thin (typically around $20 \mu \mathrm{m}$ ), it overwhelms the electrical properties of skin. Its high resistivity makes skin one of the most resistive tissues in the human body. Deeper skin layers all have much lower resistivities [17]-[19]. Electric pulses applied on the skin cause the applied voltage to be distributed between the skin layers proportionally to their resistivities (as in serially connected resistors-a circuit known as voltage divider). That means the electric field in the stratum corneum is severalfold higher than the electric field in the dermis and the viable epidermis where it stays well under electropermeabilization values, even if the applied voltage is very high. At first glance, this seems contradictory to in vivo experiments, showing a successful DNA delivery to the dermis and the viable epidermis by means of electroporation [8], [20]. However, a phenomenon we can observe from the in vivo experiments is the increase in tissue conductivity due to cell membrane electroporation [12], [13], [16]. Namely, in the areas in the tissue where the electric field exceeds a given threshold, tissue conductivity increases, consequently changing the electric field distribution. In this way, the layers underneath the stratum corneum end up being subjected to an electric field high enough for a successful tissue permeabilization. 
Furthermore, it has been shown that the electropermeabilization and, consequently, the increase in the conductivity of the stratum corneum are not homogeneous throughout the electroporated area. Molecular and ionic transport across the skin subjected to high-voltage pulses is highly localized in the so-called local transport regions (LTRs). Their size and density depend on pulse parameters such as pulse amplitude and length. It has been suggested that longer electric pulses produce larger LTRs, as the alteration of the stratum corneum structure is caused by a synergistic effect between electroporation and Joule heating, while higher pulse amplitude means higher LTR density [21]-[25]. Although more research needs to be done, skin electroporation and LTR formation using short high-voltage pulses is believed to be a nonthermal phenomenon involving structural rearrangements in skin.

Numerical modeling of the electric field and the electric current distributions inside biological systems represent an important field in the study of the effects of the electromagnetic fields on cells, tissues, and organs. A theoretical model in agreement with experimental results can be a powerful tool and can offer useful insight into the understanding of intricate biological processes taking place in the tissue during electropermeabilization. We can evaluate various electrical parameters, plan future in vivo experiments, and design electrode geometries. In this paper, we took into account the layered structure of skin and changes of its bulk electrical properties during electroporation, as observed in the in vivo experiments. In addition, the microscopical aspect of skin electropermeabilization - the presence of localized sites of increased molecular transport termed LTRs-was modeled. We used different pulse voltage amplitudes and compared the currents given by the model with measured currents obtained in vivo.

\section{Methods}

Electric field and current calculations were made by means of commercially available computer program COMSOL Multiphysics, version 3.3 (COMSOL, Los Angeles, CA, USA), based on finite element method. This method solves partial differential equations (PDEs) by dividing the model into smaller elements where the quantity to be determined is approximated with a function or is assumed to be constant throughout the element. Finite elements can be of different shapes and sizes, which allows modeling of intricate geometries. Inhomogeneities and anisotropies can also be modeled, and different excitations and boundary conditions can be applied easily. COMSOL Multiphysics is a powerful interactive environment for modeling and solving a number of scientific and engineering problems based on PDEs. With this package, it is possible to build models by defining the relevant physical quantities-such as material properties, loads, constraints, sources, and fluxes-rather than by defining the underlying equations. COMSOL Multiphysics then internally compiles a set of PDEs representing the entire model. Models can be built through a flexible graphical user interface, or by script programming in the COMSOL Script language or in the MATLAB language.

\section{A. Numerical Model of Skin Electropermeabilization From Macroscopical Aspect}

Experiments show that the conductivity of a tissue changes during electroporation [12], [13], [16]. We simulated this process with a numerical model, modeling tissue and electrode setups and pulse parameters that we used in the in vivo experiments reported previously [8]. Four layers of skin, formed into a skin fold, were modeled: stratum corneum, epidermis, dermis, and the underlying layer of fat and connective tissue. Two parallel electrodes with the distance of $4 \mathrm{~mm}$ between the plates were modeled as a boundary condition. The area of the electrodes in contact with skin was approximately $1 \mathrm{~cm} \times$ $1 \mathrm{~cm}$. In addition, a conductive gel was used in experiments in order to assure good electrical contact between the skin and the electrodes. At this point, it needs to be emphasized that electric pulses are applied on the surface where either the electrodes or conductive gel are in contact with skin. In this way, the electric pulses were applied on skin somewhat beyond the size of the electrodes (see Fig. 1). However, in the numerical model, conductive gel was not modeled as a separate layer. By using symmetry and applying appropriate boundary conditions, only one fourth of the whole geometry can be modeled, thus avoiding numerical problems due to the complexity of the model and computer memory limitations. The geometry of our numerical model is shown in Fig. 1. The thickness of the stratum corneum in the model is set larger than in real skin (approximately six times). Namely, due to the large differences in layer thicknesses numerical problems can occur, rendering the calculation impossible. To make up for the stratum corneum being modeled thicker than it is, the conductivity of this very resistive layer was also set six times higher.

When the electric field is applied to the skin fold, almost the entire voltage is on the outermost layer of the skin called the stratum corneum, due to its lowest conductivity. Namely, applying electric pulses on such a voltage divider causes the voltage to be distributed between the resistors proportionally to their resistivities. In our case, it means that almost the entire voltage rests across the highly resistive (poorly conductive) stratum corneum. However, because of the changes of bulk electrical properties of the electropermeabilized tissues, once the stratum corneum is permeabilized, the electric field "penetrates" to the layers underneath it. This process was modeled as an irreversible phase transition problem, taking into account the increase in tissue conductivity due to cell membrane electropermeabilization. Namely, when the electric field exceeds the predefined threshold, tissue conductivity increases. This change subsequently causes the change of the electric field distribution and of the corresponding current. Therefore, the final solution has to be reached iteratively. At the beginning, the electric field distribution is that of the nonpermeabilized tissue, where all the tissues involved have their initial conductivity values. In the regions where electric field exceeds the electropermeabilization threshold, the conductivity $(\sigma)$ changes according to the predefined functional dependence $\sigma(\mathrm{E})$ (see Table I). The electric field distribution is computed again with these new conductivity values. The conductivity of any given part of the tissue, once the threshold electric field is reached and exceeded, stays at 


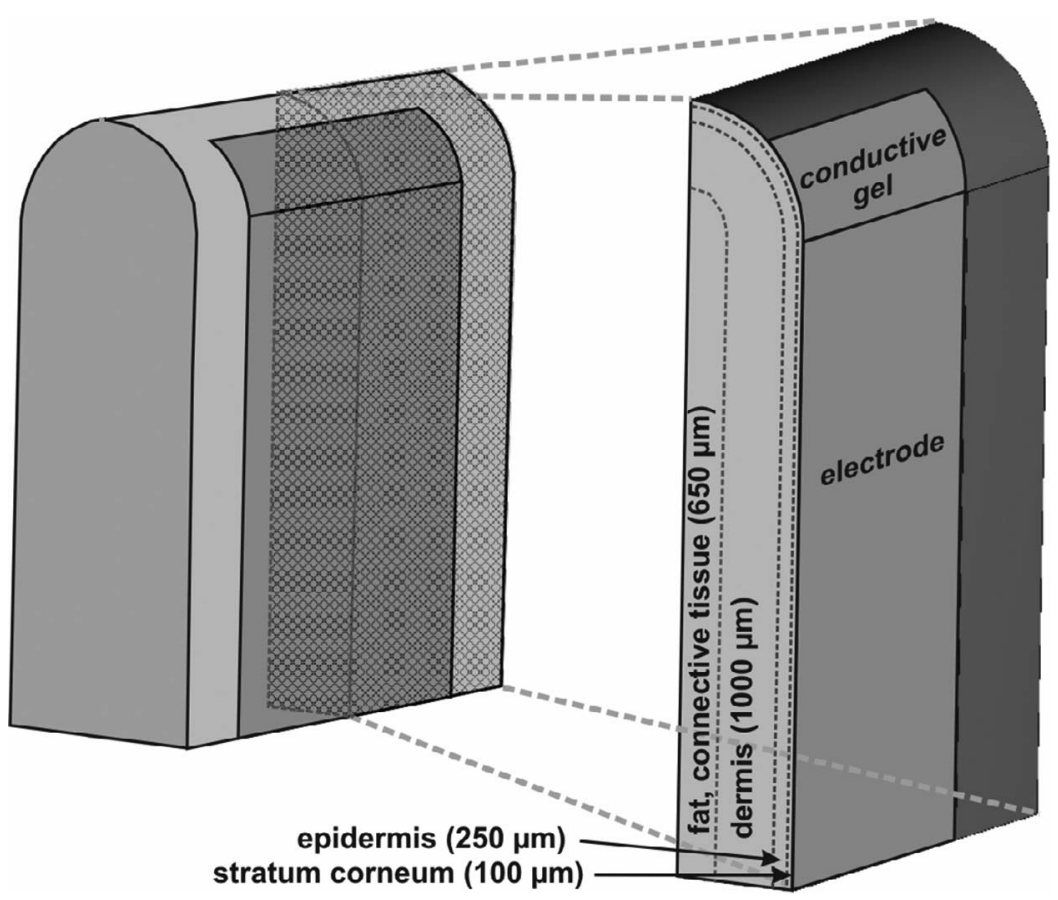

Fig. 1. Geometry of the skin fold macroscopical finite element model made in COMSOL. Only one fourth of the skin fold was modeled to avoid numerical problems and save computer time. The thickness of the stratum corneum in the model is set larger than in real skin (approximately six times). Namely, due to the large differences in layer thicknesses numerical problems can occur, rendering the calculation impossible. To make up for the stratum corneum being modeled thicker than it is, the conductivity of this very resistive layer was also set six times higher.

TABLE I

Parameters Used in the Macroscopical Numerical Model of the Electropermeabilization Process IN SKin

\begin{tabular}{l|ccc}
\hline & Subcutaneous layer & Dermis, epidermis & Stratum corneum \\
\hline$\sigma_{(\mathrm{E}<600 \mathrm{~V} / \mathrm{cm})}$ & $50 \mathrm{mS} / \mathrm{m}$ & $200 \mathrm{mS} / \mathrm{m}$ & $0.5 \mathrm{mS} / \mathrm{m}$ \\
$\sigma_{(600 \mathrm{~V} / \mathrm{cm}<\mathrm{E}<800 \mathrm{~V} / \mathrm{cm})}$ & $55 \mathrm{mS} / \mathrm{m}$ & $220 \mathrm{mS} / \mathrm{m}$ & $16.5 \mathrm{mS} / \mathrm{m}$ \\
$\sigma_{(800 \mathrm{~V} / \mathrm{cm}<\mathrm{E}<1000 \mathrm{~V} / \mathrm{cm})}$ & $68 \mathrm{mS} / \mathrm{m}$ & $270 \mathrm{mS} / \mathrm{m}$ & $60 \mathrm{mS} / \mathrm{m}$ \\
$\sigma_{(1000 \mathrm{~V} / \mathrm{cm}<\mathrm{E}<1200 \mathrm{~V} / \mathrm{cm})}$ & $103 \mathrm{mS} / \mathrm{m}$ & $410 \mathrm{mS} / \mathrm{m}$ & $178 \mathrm{mS} / \mathrm{m}$ \\
$\sigma_{(\mathrm{E}>1200 \mathrm{~V} / \mathrm{cm})}$ & $200 \mathrm{mS} / \mathrm{m}$ & $800 \mathrm{mS} / \mathrm{m}$ & $500 \mathrm{mS} / \mathrm{m}$ \\
\hline
\end{tabular}

its high value, even if the new electric field distribution should suggest otherwise (irreversible phase transition). The process is repeated until the electric field distribution reaches its steady state. That is, when there are no more areas where the electric field is above the predefined threshold. The electrical conductivity values, their changes during electroporation, and the electric field electropermeabilization thresholds were obtained from the literature and experiments [8]-[19]. It is difficult to get their exact values due to the lack of measurements and large discrepancies in the reported data; nevertheless, we used the data available to set those parameters. The conductivities used in our model were in the middle of the value ranges found in the literature.

Exactly how tissue conductivities $(\sigma)$ change with electric field (E) is yet another unknown of tissue electropermeabilization. Due to the nonuniformity of the cell size and shape in the tissue, not all the cells are permeabilized at the same time once the threshold electric field is reached. Therefore, we assumed a gradual increase of the conductivities with electric field. In our model, the conductivities were increased from their low to their high values in four steps. The conductivity steps for all the skin layers followed an exponential dependence between 600 and $1400 \mathrm{~V} / \mathrm{cm}$ of the electric field strength. Namely, the electric field threshold value needed for skin electropermeabilization was found to be approximately $400-600 \mathrm{~V} / \mathrm{cm}$. Consequently, conductivity increases of all skin layers modeled also occur above that value. The conductivity values used in our model are summarized in Table I. Although the dermis and the epidermis were modeled as separate layers, the same conductivity was assigned to both, due to the lack of conductivity data on different skin layers.

In our in vivo experiments, five different pulse amplitudes were used to permeabilize the skin: $160,280,400,520$, and $700 \mathrm{~V}$. Our finite element models were also solved for all five voltages used, and the electric currents obtained from the models were compared to corresponding experimental data. 


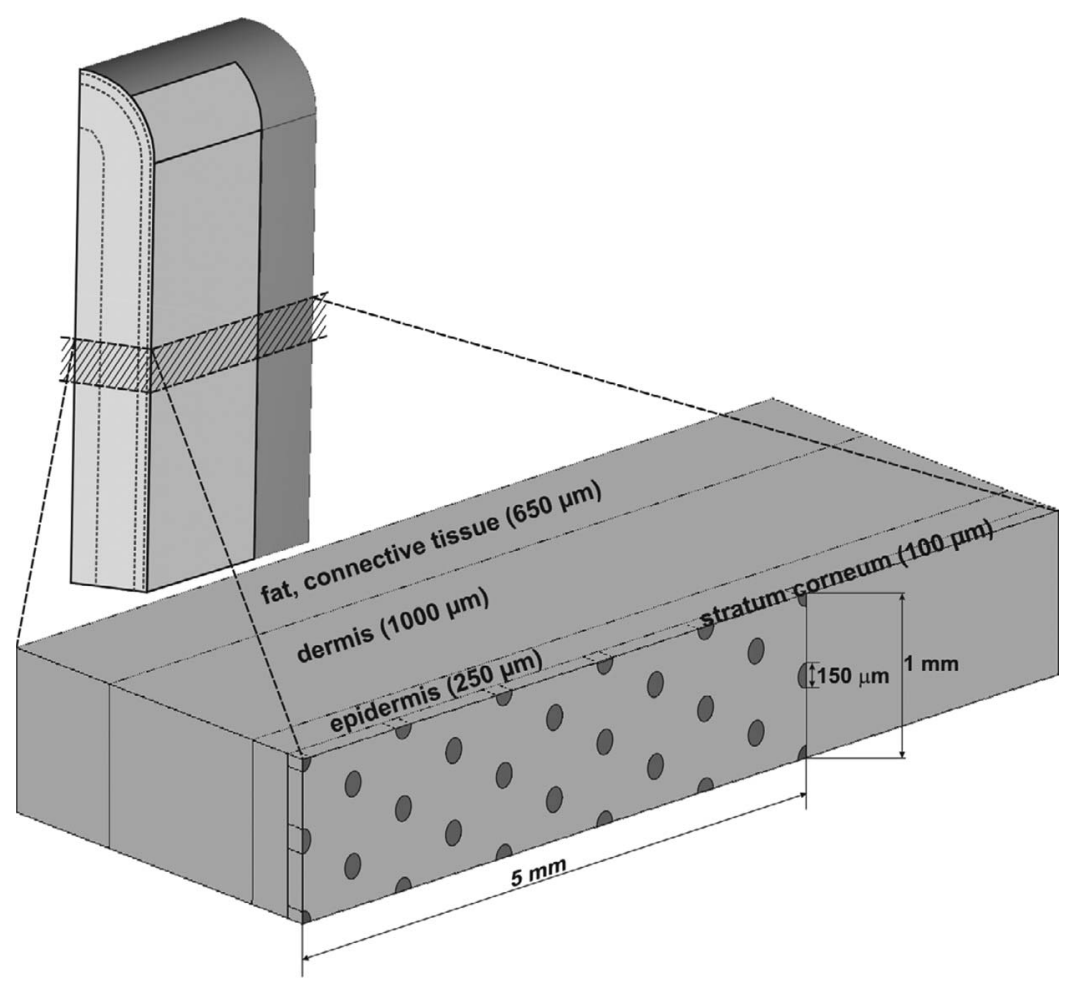

Fig. 2. Geometry of the finite element model representing a slice of a skin fold with LTRs (the geometry with 40 LTRs per $0.1 \mathrm{~cm}^{2}$ is shown). Using periodic boundary conditions, only one tenth of the macroscopical model described in the previous subchapter was modeled to avoid numerical problems and save computer time.

\section{B. Numerical Model of Skin \\ Electropermeabilization With LTRs}

It has been shown that molecular and ionic transport across skin subjected to high-voltage pulses is highly localized. The localized sites of molecular transport are called local transport regions (LTRs) [14], [21]-[25]. The authors have shown that the size of the LTR depends on pulse duration, while pulse amplitude dictates the density. LTRs are formed in the sites of the so-called stratum corneum "defects." They are further expanded by Joule heating caused by high local current density due to the drop in the resistivity of the stratum corneum inside the LTRs [14]. Taking all that into account, we made another numerical model of skin electropermeabilization, where LTRs were modeled as highly conductive structures in the stratum corneum. The results obtained from this model were compared to the results from the macroscopical model and to the in vivo data previously published [8].

In the model where LTRs were introduced, the same geometry as described in the previous subchapter was used, consisting of four layers of skin: stratum corneum, epidermis, dermis, and fat with connective tissue. The thicknesses of the skin layers were the same as in the skin fold model without LTRs; however, only a $1 \mathrm{~mm}$ slice of the skin fold was modeled, as shown in Fig. 2. Periodic boundary conditions were set as to model the whole skin fold while simplifying the otherwise intricate geometry including LTRs. The same nonlinear $\sigma(\mathrm{E})$ dependences as well as the conductivity values before and after permeabilization as in the macroscopical model were used for all the skin layers (see Table I), except for the stratum corneum. The conductivity of the nonpermeabilized stratum corneum was set to $0.0005 \mathrm{~S} / \mathrm{m}$, while the conductivity of the permeabilized areas in the stratum corneum - the LTRs-was $1.25 \mathrm{~S} / \mathrm{m}$. In our model, the process of the LTR formation itself was not described. Instead, only the steady state of the electropermeabilization process of the stratum corneum was modeled, with LTRs exhibiting highly increased conductivity, while the conductivity of the rest of the stratum corneum remained low. We assumed that the stratum corneum was already permeabilized at the beginning of the simulation, while the permeabilization of other skin layers was computed in the same way as in the macroscopical model.

The data on LTR size and density and their conductivity were taken from the literature [21]-[23]. Reported size of LTRs ranges from 50 to $600 \mu \mathrm{m}$ in diameter and their density from 5 to 90 LTRs per $0.1 \mathrm{~cm}^{2}$. Specifically, for high-voltage pulses, the size of the resulting LTRs is typically around $150 \mu \mathrm{m}$ in diameter and their density 25 to 90 LTRs per $0.1 \mathrm{~cm}^{2}$. The size of the LTRs increases with longer pulse duration, while their density is increased with increasing pulse voltage. The geometry of our model of the skin electropermeabilization taking into account LTRs gives the following values describing LTRs and stratum corneum.

1) LTR size: $150 \mu \mathrm{m}$ in diameter.

2) LTR density: 40 or 80 LTRs per $0.1 \mathrm{~cm}^{2}$. Two numerical models with LTRs in the stratum corneum were made. Namely, as LTR density increases with increasing voltage, we cannot assume the same density for the whole range of pulse amplitudes the model was solved for.

3) LTR covered surface: $7 \%$ or $14 \%$ of the stratum corneum surface beneath the electrode (according to literature, 


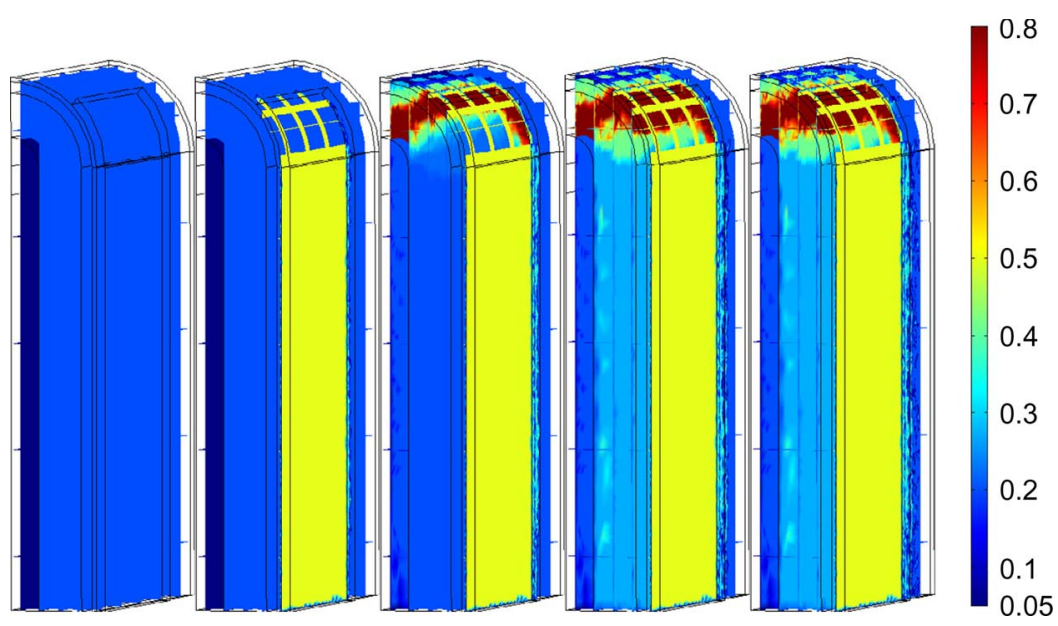

Fig. 3. Three-dimensional slice plots of tissue conductivities during the electropermeabilization process in the macroscopical numerical model. The five plots represent five stages of the process in chronological order; however, time interval between them is, in general, not uniform. The conductivity is given in siemens per meter.

the application of high-voltage pulses produces LTRs that cover around $10 \%$ of the stratum corneum surface [22], [23]).

4) Nonpermeabilized stratum corneum conductivity: $0.0005 \mathrm{~S} / \mathrm{m}$ (the same initial conductivity as used in the macroscopical model).

5) LTR conductivity: 2500 times higher than that of the stratum corneum [22], which amounts to $1.25 \mathrm{~S} / \mathrm{m}$.

6) The calculated average conductivity of the modeled stratum corneum with LTRs (the region covered by electrode): 0.09 or $0.18 \mathrm{~S} / \mathrm{m}$ (for comparison, the conductivity of the permeabilized stratum corneum in the macroscopical model, where LTRs were not modeled, was $0.5 \mathrm{~S} / \mathrm{m})$.

\section{RESULTS AND DISCUSSION}

\section{A. Numerical Model of Skin Electropermeabilization \\ From Macroscopical Aspect}

The finite element model of skin electropermeabilization was solved at five voltages used in our in vivo experiments reported previously [8]-160, 280, 400, 520, and $700 \mathrm{~V}$-in order to compare the results given by the model to the experimental results. With our numerical models, we described tissue permeabilization from the aspect of conductivity changes of the permeabilized tissues. Fig. 3 shows the evolution of the conductivity changes during electroporation in five steps, from the beginning of the process (skin layers are not permeabilized and have their initial conductivities) to the steady state of the electropermeabilization process (tissues are permeabilized to the level dictated by pulse amplitude, geometry, and tissue properties), for $400 \mathrm{~V}$ of the applied voltage between the electrodes. This voltage was chosen because it resulted in a successful gene transfection in vivo [8]. The results on all images are shown with vertical section planes, resulting in the gridded pattern throughout the model. The plots in Fig. 3 show that the first tissue to get permeabilized is the stratum corneum, as anticipated. The increased conductivity of the stratum corneum and, consequently, changed electric field distribution (see Fig. 4) enables the permeabilization of the underlying layers, the viable epidermis and the dermis, which are target layers for gene transfection. As suggested by the last plot shown in Fig. 3, the target layers are permeabilized, the level of permeabilization being the highest in the topmost part of the skin fold, where during our in vivo experiments, the plasmid was injected intradermally. This higher level of tissue permeabilization at the top of the skin fold is caused by a shorter distance between the applied voltage boundary condition (the presence of conductivity gel). Namely, higher $U / d$ ratio means higher electric field. With this in mind, we were comparing the voltages needed for a successful electropermeabilization of the skin fold as suggested by the model, with voltages achieving efficient in vivo gene transfection (results published in [8]), and good agreement was observed. In vivo experiments showed a lower expression when $160 \mathrm{~V}$ was used because the electric field in the viable epidermis and the dermis is still too low, as confirmed by the model. Similarly, the in vivo expression was lower when 520 or $700 \mathrm{~V}$ were used, due to lower cell viability caused by damaging high electric fields, which is again consistent with the results of the model, showing a very high electric field throughout the model at those voltages.

If we analyze the pulse amplitude of $400 \mathrm{~V}$ applied during our in vivo experiments, we get $1000 \mathrm{~V} / \mathrm{cm}$ voltageto-distance ratio (distance between the plates is $d=4 \mathrm{~mm}$ ) which, according to the literature [24], [26], is high enough for a successful skin permeabilization. Looking at the electric field distributions given by the model, we get similar results. Electric field distributions represented with slice plots in 3-D during the electropermeabilization process (in five steps) for the applied voltage of $400 \mathrm{~V}$ are shown in Fig. 4. The figures show the area between the electric field strength of 600 $1400 \mathrm{~V} / \mathrm{cm}$, i.e., in the range where the conductivity increase was predicted in the model (between blue and red colors). In the blue areas, the electric field was below $600 \mathrm{~V} / \mathrm{cm}$, while the red color shows the areas above $1400 \mathrm{~V} / \mathrm{cm}$ of the electric field strength. 


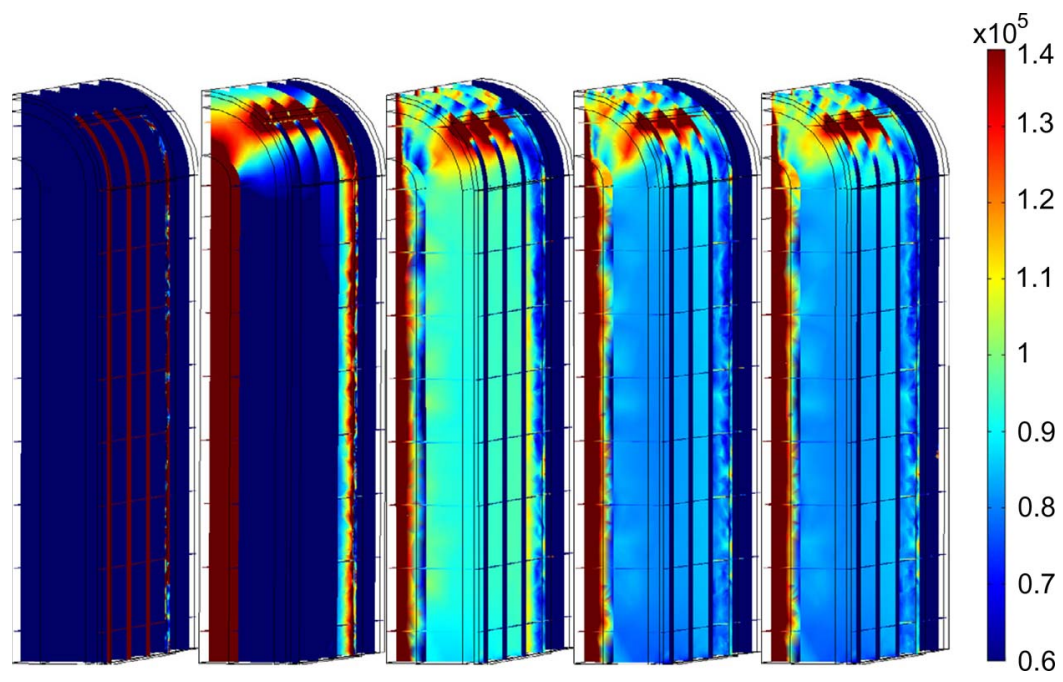

Fig. 4. Three-dimensional slice plots of the electric field distributions during the electropermeabilization process in the skin fold for the applied voltage 400 V. The five plots represent five stages of the process in chronological order; however, time interval between them is, in general, not uniform. The electric field is shown in volts per meter.

\section{B. Numerical Model of Skin \\ Electropermeabilization With LTRs}

The experiments of other researchers revealed highly localized molecular transport in skin after electroporation [14], [21]-[24]. These circularly shaped regions of increased molecular transport were termed local transport regions (LTRs). We constructed two different models of skin with two different densities of LTRs embedded in the stratum corneum (as opposed to the macroscopical model where the stratum corneum was a homogeneous structure) based on the available data on the size, density, and electrical properties of LTRs found in the literature. The geometry of the macroscopical model was used as the basis for the LTR model. However, only a slice the size of one tenth of the skin fold volume between the electrodes was modeled in the LTR model, and periodic boundary conditions were set. The layer thicknesses, the conductivities, and the $\sigma(\mathrm{E})$ dependences (except for the stratum corneum) were the same for all models. The numerical description of the stratum corneum included LTRs exhibiting highly increased conductivity, while the conductivity of the rest of the stratum corneum remained low. Only the steady state of the electropermeabilization process of the stratum corneum was modeled.

The finite element models were again solved for five voltages used in our in vivo experiments [8]. As LTR density increases with increasing pulse amplitude, the model having lower LTR density (40 LTRs per $0.1 \mathrm{~cm}^{2}$ ) was solved for 160 and $280 \mathrm{~V}$, while the one having higher LTR density ( 80 LTRs per $0.1 \mathrm{~cm}^{2}$ ) was solved for 400,520 , and $700 \mathrm{~V}$. The data on LTR densities were taken from the literature [21]-[23]. The reported LTR densities are between 25 and 90 LTRs per $0.1 \mathrm{~cm}^{2}$ for short high-voltage pulses. The LTR densities of our models (40 and 80 LTRs per $0.1 \mathrm{~cm}^{2}$ ) are inside that range. At this stage, only the steady state of LTR formation was modeled. Namely, LTRs are formed as a result of the application of electric pulses on skin and are expanded by Joule heating caused by high local current density occurring because of the drop in the resistivity of the stratum corneum inside the LTRs. In the future, a numerical model including the thermal aspect of LTR formation and expansion will be made using multiphysics modeling.

Again, the conductivity changes and the electric field distributions during electroporation for the voltage of $400 \mathrm{~V}$ (resulting in a successful gene transfection in vivo) were chosen to be presented, for easier comparison with the macroscopical model. Fig. 5 shows the evolution of the conductivity changes in the LTR model during electroporation in four steps. Looking at the last conductivity plot, it seems that the inclusion of the highly conductive areas in the stratum corneum also leads to a nearly homogeneous permeabilization of the viable epidermis and the dermis, even though the conductivity increase in the stratum corneum is not homogeneous.

Fig. 6 shows the electric field distributions represented with slice plots in 3-D during the electropermeabilization process (in four steps) for the applied voltage of $400 \mathrm{~V}$. The area between the electric field strength of $600-1400 \mathrm{~V} / \mathrm{cm}$ (the range where the conductivity increase was predicted in the model) is again presented with the color scale between blue and red. In the uniformly blue areas, the electric field was below the $600 \mathrm{~V} / \mathrm{cm}$, while the red color shows the areas above $1400 \mathrm{~V} / \mathrm{cm}$ of the electric field. Comparing the LTR model with the macroscopical model, $400 \mathrm{~V}$ seems to result in somewhat lower level of tissue permeabilization in the former. Namely, comparing Figs. 3 and 5, the conductivity increase is lesser in the LTR model. In addition, the electric field distribution is shifted to somewhat lower levels when LTRs are modeled in the stratum corneum (Figs. 4 and 6).

\section{Numerical Models_In Vivo Experiments Electric Current Comparison}

During the in vivo experiments, the voltage between the electrodes and the current through the skin fold were measured. The numerical models of skin electropermeabilization were solved at all five voltages used, and the electric currents given by the models were compared to our experimental data. Fig. 7 


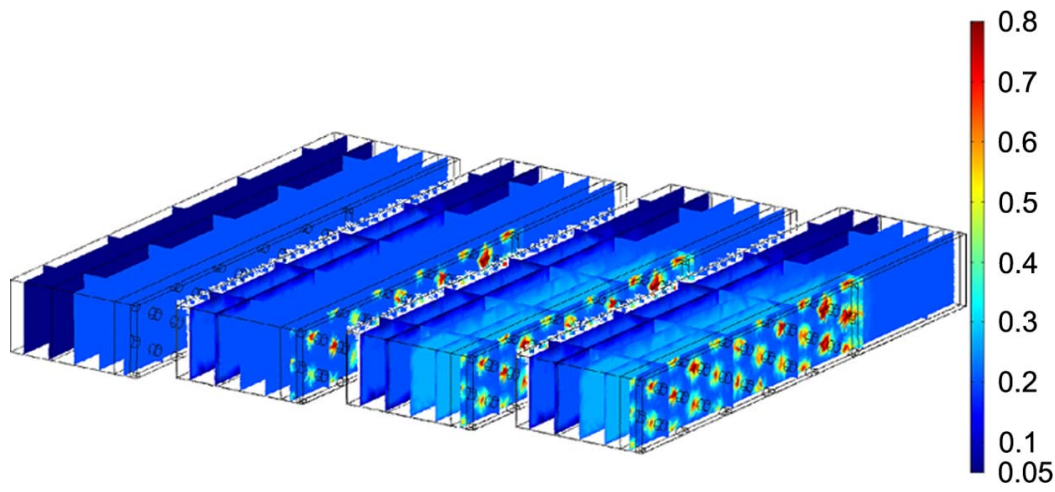

Fig. 5. Three-dimensional slice plots of the conductivity distributions during the electropermeabilization process in the LTR model for the applied voltage $400 \mathrm{~V}$. The four plots represent four stages of the process in chronological order; however, time interval between them is, in general, not uniform. The conductivity is given in siemens per meter.

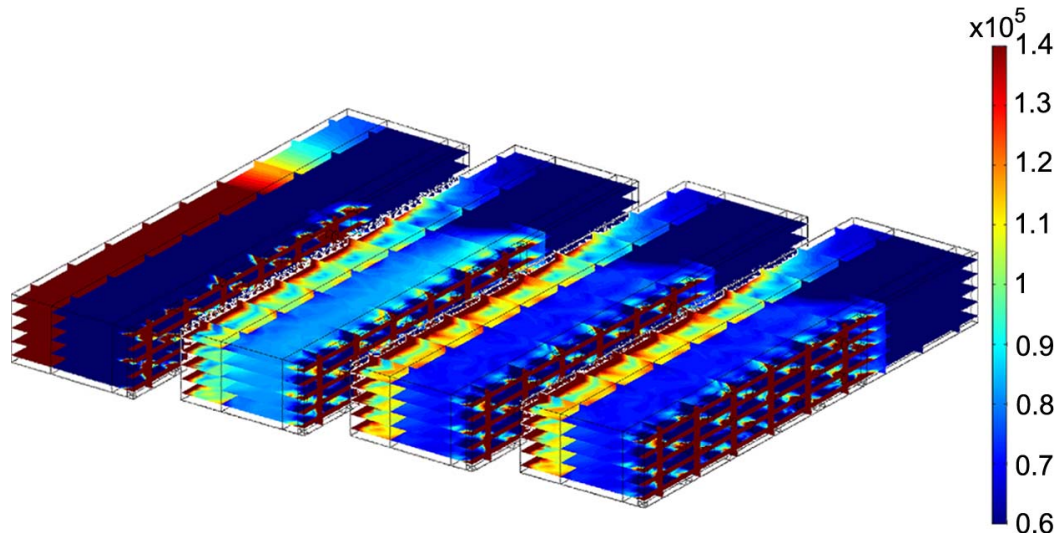

Fig. 6. Three-dimensional slice plots of the electric field distributions during the electropermeabilization process in the LTR model for the applied voltage $400 \mathrm{~V}$. The four plots represent four stages of the process in chronological order; however, time interval between them is, in general, not uniform. The electric field is shown in volts per meter.

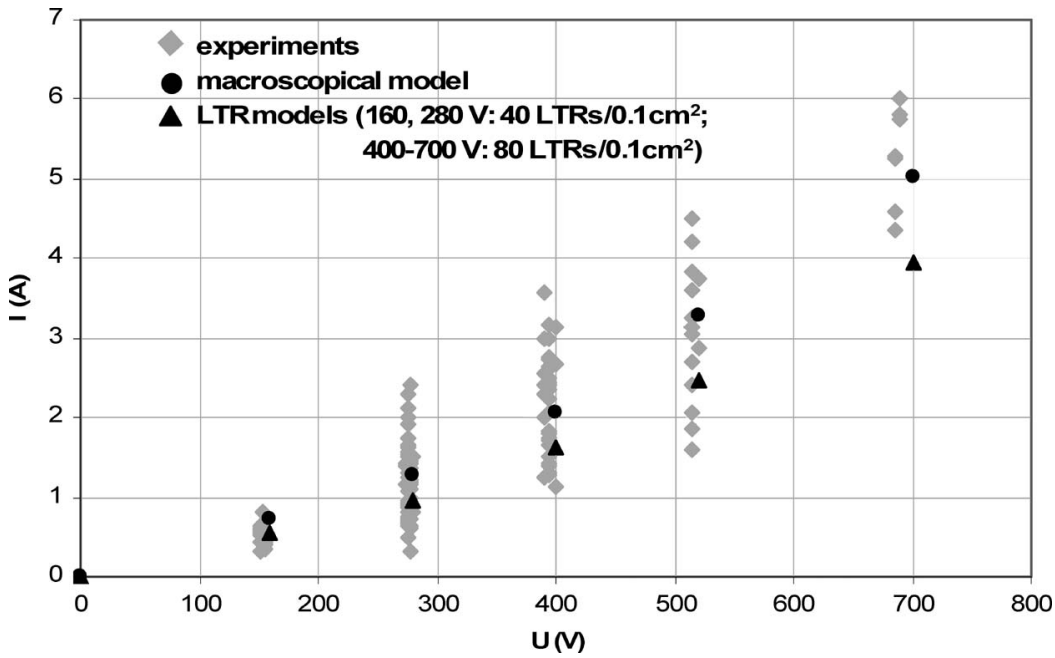

Fig. 7. Currents measured during the pulse (gray diamonds), compared to the currents given by the macroscopical model (black circles) and those given by the LTR model (black triangles) with respect to the applied voltages.

shows the currents of both models as compared to the currents measured in vivo during the pulse. A good agreement can be observed in the current-voltage dependence between the in vivo data and the output of the macroscopical model.

Comparing electric currents at the end of the electropermeabilization process, the currents given by the LTR model are shifted toward lower values by a factor of 1.3 throughout the entire pulse amplitude range modeled, when compared to the macroscopical model. The reason for this discrepancy can be attributed to different average conductivities of the permeabilized stratum corneum of both models. Namely, in the macroscopical model, the permeabilized part of the stratum 
corneum (mainly underneath the electrodes and the conductive gel) has the value of $0.5 \mathrm{~S} / \mathrm{m}$. As opposed to that, the average conductivity of the permeabilized part of the stratum corneum in the LTR model (with embedded highly conductive LTRs in the highly resistive stratum corneum) amounts to $0.09 \mathrm{~S} / \mathrm{m}$ (for LTR density of 40 LTRs per $0.1 \mathrm{~cm}^{2}$ ) or to $0.18 \mathrm{~S} / \mathrm{m}$ (for LTR density of 80 LTRs per $0.1 \mathrm{~cm}^{2}$ ). This discrepancy, together with the nonhomogeneous structure of the stratum corneum with LTRs, leads to the lower permeabilization of the underlying epidermis and dermis. Still, the results of the LTR model are well in the range of the macroscopical model and the in vivo experiments. Furthermore, the current-voltage dependence of the LTR model exerts similar nonlinearity as the one of the macroscopical model. This fact confirms the need to construct different models for each applied voltage, with different number of LTRs. Namely, as reported in the literature, the number of the LTRs increases with increasing voltage [21]. Indeed, if we try using a model with the same LTR density for the whole range of pulse amplitudes, the current/voltage dependence becomes more linear. The currents given by the LTR model, when compared to the macroscopical model, are shifted toward lower values by a varying factor of $1.3-1.5$, the lower and the higher values corresponding to pulse amplitude of 160 and $700 \mathrm{~V}$, respectively.

\section{CONCLUSION}

Skin is, due to its size and accessibility, an attractive target tissue for applications such as transdermal drug delivery and in vivo gene delivery. Its protective function and low permeability can be temporarily breached using electroporation, thereby creating aqueous pathways across otherwise nonpermeable lipid-based structure. Various in vivo experiments performed up to date show a successful transdermal drug delivery or DNA delivery to the dermis and the viable epidermis by means of electroporation. Theoretically, the ratios of the conductivities of skin layers suggest that the highest voltage rests across the stratum corneum, while the electric field in the layers below is too low for a successful permeabilization. However, the experiments also suggest changes in tissue conductivity during electroporation, depending on the electric field the tissue is subjected to. We constructed a numerical model describing the nonlinear process of tissue conductivity changes during electroporation due to tissue permeabilization, using finite element method. The output of the model was compared to the current and the voltage measured during in vivo experiments [8], and a good agreement was obtained. In addition, comparing the voltages needed for a successful electropermeabilization of the skin fold as suggested by the model, with voltages achieving good in vivo gene transfection in our in vivo experiments, good agreement can be observed. Finally, a comparison of our results with findings on skin electropermeabilization published by other authors [24], [26] showed that the voltage amplitudes suggested by the model are also well in the range of the voltage amplitudes reported to cause skin permeabilization.

Furthermore, the experiments of other researchers revealed highly localized molecular transport in skin after electroporation [14], [21]-[25]. We thus made a model of skin with LTRs embedded in the stratum corneum, based on the data on the size, density, and electrical properties of LTRs found in the literature. The conductivity increase and the electric fields given by the LTR model are somewhat lower when compared to the results of the macroscopical model and the in vivo experiments. Comparing electric currents at the end of the electropermeabilization process, the currents given by the LTR model are also shifted toward somewhat lower values. As the size, density, and the conductivity of LTRs were taken from the literature, where different electroporation protocols were used than in our in vivo experiments, the parameters of the LTRs formed because of electroporation in our experiments might have been different. Namely, trains of exponentially decaying pulses were used by the researchers reporting the visualization of the LTRs, while only one square electroporative pulse was used in our experiments. In addition, the tissue-electrode geometry was different, and more importantly, heat-separated human cadaver epidermis was used in their experiments. None the less, the electric field distributions and the electric currents of the LTR model are well in the range of those of the macroscopical model and the in vivo experiments. As the electroporation protocols and circumstances of our in vivo experiments are not directly comparable to the experiments of the researchers using fluorescence microscopy to localize LTRs, we can accept this as a good agreement of the results.

Some simplifications of the geometry of the numerical models were necessary due to its complexity. First, the thickness of the stratum corneum in the model was set larger than in real skin (six times thicker). Although its conductivity was also set six times higher, an error was introduced. In addition, not enough skin fold volume was modeled at the base of the skin fold, thus the natural electric current flow was somewhat restrained. We needed to accept this simplification, since the large number of the finite elements and the degrees of freedom of the model make the computation more complicated. Furthermore, with the periodic boundary conditions set on the boundaries of the skin fold slice with LTRs, an infinite array of slices was modeled. An error was thus made, as the electric field distribution around the borders of the electrodes differs from the distribution in the tissue situated well in the middle of the electrode. However, the error was small (on the order of 5\% or less). In addition, data on conductivity changes during the electropermeabilization are very scarce, most often nonexistent. Namely, the subject of tissue conductivity changes due to electroporation is still a rather unexplored field. Due to different measuring conditions, measuring techniques, and animal species used by different researchers, large discrepancies can be found in the reported data on tissue conductivities.

Nevertheless, we used the available data to describe the mechanism of the nonlinear process of skin electropermeabilization from the aspect of bulk conductivity changes and the presence of the LTRs in the permeabilized stratum corneum. In this way, the observations derived from various in vivo experiments by different authors were confirmed theoretically. Such numerical models, further improved and validated by experiments, can be used for the simulation of permeabilization process in skin, as well as other tissues. They allow predicting the outcome of pulse delivery before the treatment and help us 
in optimizing/choosing the most efficient protocols and pulse parameters. Such an approach can also assist us in the development of electrodes and optimizing their placement with respect to target tissue in both electrogene transfer and transdermal drug delivery.

\section{REFERENCES}

[1] M. R. Prausnitz, "Reversible skin permeabilization for transdermal delivery of macromolecules," Crit. Rev. Ther. Drug Carr. Syst., vol. 14, no. 4, pp. 455-483, 1997.

[2] B. W. Barry, "Novel mechanisms and devices to enable successful transdermal drug delivery," Eur. J. Pharm. Sci., vol. 14, no. 2, pp. 101-114, Sep. 2001.

[3] J. J. Drabick, J. Glasspool-Malone, A. King, and R. W. Malone, "Cutaneous transfection and immune responses to intradermal nucleic acid vaccination are significantly enhanced by in vivo electropermeabilization," Molec. Ther., vol. 3, no. 2, pp. 249-255, Feb. 2001.

[4] L. Zhang, G. Widera, and D. Rabussay, "Enhancement of the effectiveness of electroporation-augmented cutaneous DNA vaccination by a particulate adjuvant," Bioelectrochemistry, vol. 63, no. 1/2, pp. 369-373, Jun. 2004.

[5] M. R. Prausnitz, "A practical assessment of transdermal drug delivery by skin electroporation," Adv. Drug Deliv. Rev., vol. 35, no. 1, pp. 61-76, Jan. 1999.

[6] A.-R. Denet, R. Vanbever, and V. Préat, "Skin electroporation for transdermal and topical delivery," Adv. Drug Deliv. Rev., vol. 56, no. 5, pp. 659-674, Mar. 2004.

[7] E. Neumann, S. Kakorin, and K. Toensing, "Fundamentals of electroporative delivery of drugs and genes," Biochim. Biophys. Acta, vol. 48, no. 1, pp. 3-16, Feb. 1999

[8] N. Pavšelj and V. Préat, "DNA electrotransfer into the skin using a combination of one high- and one low-voltage pulse," J. Control. Release, vol. 106, no. 3, pp. 407-415, Sep. 2005.

[9] C. Gabriel, S. Gabriel, and E. Corthout, "The dielectric properties of biological tissues: I. Literature survey," Phys. Med. Biol., vol. 41, no. 11, pp. 2231-2249, Nov. 1996.

[10] S. Gabriel, R. W. Lau, and C. Gabriel, "The dielectric properties of biological tissues: II. Measurements in the frequency range $10 \mathrm{~Hz}$ to 20 GHz," Phys. Med. Biol., vol. 41, no. 11, pp. 2251-2269, Nov. 1996.

[11] S. A. Gallo, A. R. Oseroff, P. G. Johnson, and S. W. Hui, "Characterization of electric-pulse-induced permeabilization of porcine skin using surface electrodes," Biophys. J., vol. 72, no. 6, pp. 2805-2811, Jun. 1997.

[12] U. Pliquett, R. Langer, and J. C. Weaver, "Changes in the passive electrical properties of human stratum corneum due to electroporation," Biochim. Biophys. Acta, vol. 1239, no. 2, pp. 111-121, Nov. 1995.

[13] A. Jadoul, J. Bouwstra, and V. Préat, "Effects of iontophoresis and electroporation on the stratum corneum. Review of the biophysical studies," Adv. Drug Deliv. Rev., vol. 35, no. 1, pp. 89-105, Jan. 1999.

[14] U. Pliquett, "Mechanistic studies of molecular transdermal transport due to skin electroporation," Adv. Drug Deliv. Rev., vol. 35, no. 1, pp. 41-60, Jan. 1999.

[15] U. Pliquett and J. C. Weaver, "Electroporation of human skin: Simultaneous measurement of changes in the transport of two fluorescent molecules and in the passive electrical properties," Bioelectrochem. Bioenerg., vol. 39, no. 1, pp. 1-12, Feb. 1996.

[16] M. R. Prausnitz, V. G. Bose, R. Langer, and J. C. Weaver, "Electroporation of mammalian skin: A mechanism to enhance transdermal drug delivery," Proc. Natl. Acad. Sci. USA, vol. 90, no. 22, pp. 10 504-10 508, Nov. 1993.

[17] T. Yamamoto and Y. Yamamoto, "Electrical properties of the epidermal stratum corneum," Med. Biol. Eng., vol. 14, no. 2, pp. 151-158, Mar. 1976.

[18] T. Yamamoto and Y. Yamamoto, "Dielectric constant and resistivity of epidermal stratum corneum," Med. Biol. Eng., vol. 14, no. 5, pp. 494-500, Sep. 1976
[19] Y. A. Chizmadzhev, A. V. Indenbom, P. I. Kuzmin, S. V. Galichenko, J. C. Weaver, and R. O. Potts, "Electrical properties of skin at moderate voltages: Contribution of appendageal macropores," Biophys. J., vol. 74, no. 2, pp. 843-856, Feb. 1998.

[20] N. Pavšelj, V. Préat, and D. Miklavčič, "A numerical model of skin electropermeabilization based on in vivo experiments," Ann. Biomed. Eng., vol. 35, no. 12, pp. 2138-2144, Dec. 2007.

[21] R. Vanbever, U. F. Pliquett, V. Préat, and J. C. Weaver, "Comparison of the effects of short, high-voltage and long, medium-voltage pulses on skin electrical and transport properties," J. Control. Release, vol. 60, no. 1, pp. 35-47, Jun. 1999

[22] U. Pliquett and C. Gusbeth, "Surface area involved in transdermal transport of charged species due to skin electroporation," Bioelectrochemistry, vol. 65, no. 1, pp. 27-32, Dec. 2004.

[23] U. F. Pliquett, R. Vanbever, V. Préat, and J. C. Weaver, "Local transport regions (LTRs) in human stratum corneum due to long and short 'high voltage' pulses," Bioelectrochem. Bioenerg., vol. 47, no. 1, pp. 151-161, Nov. 1998.

[24] J. C. Weaver, T. E. Vaughan, and Y. A. Chizmadzhev, "Theory of electrical creation of aqueous pathways across skin transport barriers," Adv. Drug Deliv. Rev., vol. 35, no. 1, pp. 21-39, Jan. 1999.

[25] U. Pliquett, S. Gallo, C. Gusbeth, E. Neumann, and S. W. Hui, "Local and transient structural changes in stratum corneum at high electric fields: Contribution of Joule heating," Bioelectrochemistry, vol. 67, no. 1, pp. 37 46, Sep. 2005.

[26] N. Pavšelj, Z. Bregar, D. Cukjati, D. Batiuskaite, L. M. Mir, and D. Miklavčič, "The course of tissue permeabilization studied on a mathematical model of a subcutaneous tumor in small animals," IEEE Trans. Biomed. Eng., vol. 52, no. 8, pp. 1373-1381, Aug. 2005.

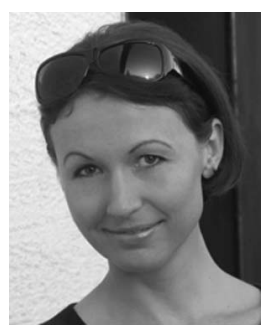

Nataša Pavšelj was born in Ljubljana, Slovenia, in 1974. She received the B.Sc., M.Sc., and Ph.D degrees in electrical engineering from the University of Ljubljana, Ljubljana.

She is currently with the Faculty of Electrical Engineering, University of Ljubljana. Her main research interests are in the field of electroporation, including finite element numerical modeling of electric field distribution in different biological tissue setups (subcutaneous tumors, skin fold) and comparison of the theoretical results with the experimental work In the last couple of years, her research work is specifically oriented on understanding and describing the process of skin electropermeabilization, mostly involving numerical modeling as well as in vivo experimenting.

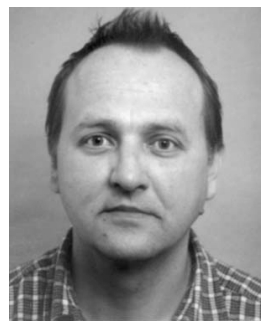

Damijan Miklavčič was born Ljubljana, Slovenia, in 1963. He received the Ph.D. degree in electrical engineering from the University of Ljubljana, Ljubljana.

$\mathrm{He}$ is currently a Professor with the Faculty of Electrical Engineering and the Head of the Laboratory of Biocybernetics, University of Ljubljana. He is active in the field of biomedical engineering. His interest in the last years focuses on electroporationassisted drug and gene delivery, including cancer treatment by means of electrochemotherapy, tissue oxygenation, and modeling. 